\title{
Non-parametric Multiple-Antenna Blind Spectrum Sensing by Predicted Eigenvalue Threshold
}

\author{
K. Hassan*§, R. Gautier ${ }^{\dagger \ddagger}$, I. Dayoub ${ }^{\S}$, E. Radoi ${ }^{\dagger \ddagger}$ and M. Berbineau* \\ ${ }^{*}$ Univ Lille Nord de France, F-59000 Lille, IFSTTAR, LEOST, F-59650 Villeneuve d'Ascq \\ $\dagger$ Université Européenne de Bretagne, France \\ $\ddagger$ Université de Brest ; CNRS, UMR 3192 Lab-STICC, ISSTB \\ 6 avenue Victor Le Gorgeu, CS 93837, 29238 Brest cedex 3, France \\ $\S$ Univ Lille Nord de France, F-59000 Lille, IEMN, DOAE, F-59313 Valenciennes
}

\begin{abstract}
In this paper, we consider the problem of sensing a primary user in a cognitive radio network by employing multiple antennas at the secondary user. Among the many spectrumsensing methods, the predicted eigenvalue threshold (PET) based method is a promising non-parametric blind method that can reliably detect the primary users without any prior information. Then, a simplified PET sensing method, which needs to compare only one eigenvalue to its threshold, is introduced. Compared with the original PET sensing algorithm, the simplified algorithm significantly reduces the computational complexity without any loss in performance. A performance comparison between the proposed method and other existing methods is provided.

Index Terms-cognitive radio, spectrum sensing, multipleantenna, predicted eigenvalue threshold, random matrix theory.
\end{abstract}

\section{INTRODUCTION}

Recently, the rapid growth in wireless communications has contributed to a huge demand on the deployment of new wireless services. However, recent research published by the Federal Communications Commission (FCC) [1] shows that the traditional static frequency allocation policy is not efficient and results in poor spectrum utilization. The dramatic increase in the demand for radio spectrum and the actual low spectral efficiency has spurred the development of a next generation wireless technology referred to as cognitive radio (CR). An early work by Mitola introducing the concept of CR is [2].

Ultimately, a CR device (secondary user) must be aware of its radio environment and capable of detecting the licensed users, also known as primary users (PUs), i.e. a CR must identify the unoccupied frequency bands, called white spaces. Sensing the spectrum and dynamically accessing the white spaces will significantly improve the spectrum utilization efficiency. This ability to detect the white spaces in the spectrum band of interest by the secondary user (SU) is usually called spectrum sensing. In order to avoid interference with PUs, the SU must quickly and robustly sense which parts of the relevant spectrum are available or not. For instance, the upcoming CR based standard IEEE 802.22, which is a wireless regional network standard (WRAN), requires the detector to sense a $\mathrm{PU}$ at a signal-to-noise ratio (SNR) of at least $-22 \mathrm{~dB}$. At this power level, the probability of detection must be higher than 0.9 , while the probability of false alarm is lower than 0.1
[3]. Obviously, Spectrum sensing will be the backbone of any autonomous CR.

Matched-filtering is known as the optimum method for detecting the PUs [4]. However, there should be a priori information about the PU such known preambles, midambles, pilots etc. The performance of energy detector (ED) based sensing is limited since it is very sensitive to noise variations. On the other hand, the energy detector is simple, non coherent and does not need any knowledge on the primary users signals [5], [6]. Cyclostationarity-based detectors provide higher performance than energy detectors and are more robust to noise level variations [7]. But they require prior knowledge on the cyclic frequencies of the PU's signal. Without this prior knowledge, the cyclostationarity-based detectors have a large complexity [8].

Multiple-antenna systems have been widely deployed to improve the transmission reliability in wireless communications. In a cognitive radio network, multiple-antenna SUs are beneficial not only for a reliable communications but also to improve the performance of spectrum sensing. The multiple-antenna techniques are employed to exploit the spatial correlations of multiple received signals. The maximum ratio combining, the equal gain combining and the selection combining techniques are applied to better sense the spectrum [9], [10]. These methods are ED based and suffers of the noise uncertainty. To overcome this difficulty, the authors in [11] proposed the optimally combined energy detection (OCED) method based on the principle of maximizing the SNR. The OCED is approximated by the blindly combined energy detection (BCED) [11]. These methods, unlike ED, does not need noise power estimation and overcomes ED's sensibility to noise uncertainty. Some methods that employ the structure of the signal sample covariance matrix exist in the literature. For instance, we mention the covariance absolute value (CAV) and the covariance Frobenius norm (CFN) methods [12]. Recently, some methods based on the eigenvalues analysis of covariance matrix had emerged. The authors in [13] introduced the maximum-minimum eigenvalue (MME) and the energy with minimum eigenvalue (EME) detectors .

It was shown in [14], that the test statistic based on the generalized likelihood ratio test (GLRT), when all parameters 
are completely unknown, is the arithmetic-to-geometric mean (AGM) of the eigenvalues of the sample covariance matrix. Actually, this is a sphericity test. Even though it is effective, the AGM detector does not fully exploit the received signal structure. Therefore, a new GLRT detector was proposed in [15], [16]. The test statistic of the proposed detector is given by the ratio of the largest eigenvalue to the average of eigenvalues of the sample covariance matrix of the received signal. This GRLT was developed for a single PU signal through memoryless channel. Blind spectrum sensing with no knowledge about the signal and the noise level at the receiver has been studied in [17] based on information-theoretic criteria (ITC).

In this paper, we introduce a non-parametric blind spectrum sensing method based on the predicted eigenvalue threshold (PET) originally introduced in [18]. This method was employed to detect the number of communications sources. Here, the PET method is applied for spectrum sensing, since it is a special case of source number detection problem. Thereafter, the PET method is simplified to reduce the complexity with no performance loss. The simplified PET (SPET) generalizes the GLRT based test statistic proposed in [15], [16] to the case of multiple PUs through a channel with memory. A performance analysis based on new results in random matrix theory is provided.

The remainder of the paper is organized as follows: Section II defines the system model and introduces the different assumptions. Section III presents the PET detection algorithm. This algorithm is simplified in section IV. Section V provides the performance analysis of the proposed method. The results and algorithm performance evaluation are presented in section VI. Finally, conclusions and perspectives of the research work are presented in section VII.

\section{SySTEM MOdEL}

We assume that there are $M \geq 1$ antennas at the SU. There are two hypotheses:

$\mathcal{H}_{0}$, absence of signals, and $\mathcal{H}_{1}$, at least one signal is present.

In the presence of $P$ primary users $\left(\mathrm{PU}_{j}, 1 \leq j \leq P\right)$, the $M \times 1$ observation vector at the receiver is expressed as:

$$
\mathbf{x}(n)=\sum_{j=1}^{P} \sum_{k=0}^{C_{j}} \mathbf{h}_{j}(k) s_{j}(n-k)+\mathbf{w}(n), \quad n=1,2, \cdots
$$

where $\mathbf{w}(n)=\left[w_{1}(n), \ldots, w_{M}(n)\right]^{T}$ is the $M \times 1$ received additive white Gaussian noise vector with zero-mean and variance $\sigma_{w}^{2}$. The order of the channel between $\mathrm{PU}_{j}$ and each antenna is $C_{j}$. The vector $\mathbf{h}_{j}(n)=\left[h_{1 j}(n), \ldots, h_{M j}(n)\right]^{T}$ represents the channel among $\mathrm{PU}_{j}$ and all the antennas; i.e. $h_{i j}(n)$ is the $n^{t h}$ tap of the channel response between $\mathrm{PU}_{j}$ and the $i^{\text {th }}$ antenna.

Let us consider $L$ consecutive samples and define the corresponding signal/noise vectors:

$$
\begin{aligned}
\mathbf{x}_{L}(n) & =\left[\mathbf{x}^{T}(n), \mathbf{x}^{T}(n-1), \ldots, \mathbf{x}^{T}(n-L+1)\right]^{T} \\
\mathbf{s}_{L}(n) & =\left[\mathbf{s}_{1}^{T}(n), \mathbf{s}_{2}^{T}(n), \ldots, \mathbf{s}_{P}^{T}(n)\right]^{T} \\
\mathbf{w}_{L}(n) & =\left[\mathbf{w}^{T}(n), \mathbf{w}^{T}(n-1), \ldots, \mathbf{w}^{T}(n-L+1)\right]^{T}
\end{aligned}
$$

where $\mathbf{s}_{j}^{T}(n)=\left[s_{j}(n), s_{j}(n-1), \ldots, s_{j}\left(n-C_{j}-L+1\right)\right]$ and $L$ is called the smoothing factor. A similar system model was used in [13], [19] and the system is expressed in matrix form as

$$
\mathbf{x}_{L}(n)=\mathbf{H s}_{L}(n)+\mathbf{w}_{L}(n)
$$

where $\mathbf{H}$ is an $M L \times(C+P L)$ matrix and $C=\sum_{j=1}^{P} C_{j}$. Defining the $M L \times\left(C_{j}+L\right)$ matrix $\mathbf{H}_{j}$ as

$$
\mathbf{H}_{j}=\left[\begin{array}{cccccc}
\mathbf{h}_{j}(0) & \cdots & \cdots & \mathbf{h}_{j}\left(C_{j}\right) & \cdots & 0 \\
\vdots & \ddots & & & \ddots & \vdots \\
0 & \cdots & \mathbf{h}_{j}(0) & \cdots & \cdots & \mathbf{h}_{j}\left(C_{j}\right)
\end{array}\right],
$$

$\mathbf{H}$ is expressed as $\mathbf{H}=\left[\mathbf{H}_{1}, \mathbf{H}_{2}, \ldots, \mathbf{H}_{P}\right]$.

The covariance matrix of the received signal, set to as $\mathcal{X}=$ $\mathbf{E}\left[\mathbf{x}_{L} \mathbf{x}_{L}^{H}\right]$, gives

$$
\mathcal{X}=\mathbf{H} \mathcal{S} \mathbf{H}^{H}+\sigma_{w}^{2} \mathbf{I}_{M L}
$$

where $(*)^{H}$ represents the Hermitian transpose and $\mathcal{S}=$ $\mathbf{E}\left[\mathbf{s}_{L} \mathbf{s}_{L}{ }^{H}\right]$ is assumed to be of full rank. Let $\lambda_{1} \geq \cdots \geq \lambda_{M L}$ denote the eigenvalues of $\mathcal{X}$. The received signal covariance matrix is usually unknown. The sample covariance matrix is employed to overcome this difficulty, and is given by

$$
\mathcal{X}_{N}=\frac{1}{N} \sum_{k=1}^{N} \mathbf{x}_{L}(k)\left(\mathbf{x}_{L}(k)\right)^{H}
$$

where $N$ is the number of observed samples. The estimated eigenvalues are $\ell_{1}, \cdots, \ell_{M L}$ such as $\ell_{1} \geq \cdots \geq \ell_{M L}$.

\section{Predicted Eigenvalues Threshold (PET)}

\section{A. Mathematical Preliminaries}

The rank of the part of the covariance matrix that represents the signal (i.e., $\mathbf{H} \mathcal{S} \mathbf{H}^{H}$ ) is $C+P L$. Hence, the lowest eigenvalue of $\mathcal{X}$ is equal to $\sigma_{w}^{2}$ and its multiplicity order is equal to $(M-P) L-C$ [19]. By applying the eigenvalue decomposition, the matrix $\mathcal{X}$ has a diagonal form,

$$
\mathbf{U}^{H} \mathcal{X} \mathbf{U}=\operatorname{diag}\left(\vartheta_{1}, \cdots, \vartheta_{C+P L}, 0, \cdots, 0\right)+\sigma_{w}^{2} \mathbf{I}_{M L}
$$

in the basis $\mathbf{U}$, where $\vartheta_{1} \geq \vartheta_{2} \cdots \geq \vartheta_{C+P L}>0$. Obviously, $\lambda_{k}=\vartheta_{k}+\sigma_{w}^{2}$ for $1 \leq k \leq C+P L$. This result requires that the matrix $\mathbf{H}$ is overdetermined, i.e.,

$$
L>\frac{C}{M-P} .
$$

For simplicity, we define $q=M L$ for the rest of the paper.

Theorem 1: Suppose $N \mathcal{X}_{N}$ has the complex Wishart distribution $\mathbf{W}_{q}(N, \mathcal{X})$, and the eigenvalues of $\mathcal{X}_{N}$ and $\mathcal{X}$ are $l_{1} \geq \cdots \geq \ell_{q}$ and $\lambda_{1} \geq \cdots \geq \lambda_{k}=\cdots=\lambda_{q}=\lambda$ 
respectively. The limiting distribution of $\ell_{k}^{a v}$ the average of the lowest $q-k$ eigenvalue, as $N \rightarrow \infty$, is

$$
(N(q-k))^{(1 / 2)}\left(\ell_{k}^{a v}-\lambda\right) / \lambda \stackrel{\text { dist }}{\longrightarrow} \mathcal{N}(0,1) .
$$

In the absence of signal, the matrix $N \mathcal{X}_{N}$ follows a Wishart complex distribution, i.e. $N \mathcal{X}_{N} \backsim \mathbf{W}_{q}(N, \mathcal{X})$.

Based on that, the authors in [18] proposed an upper bound for each eigenvalue of the noise subspace. The upper threshold of $\ell_{k}$ is predicted as

$$
\ell_{k}^{u p}=\underbrace{\left[\left(m_{k}+1\right) \frac{1+t\left[N\left(m_{k}+1\right)\right]^{1 / 2}}{1-t\left(N \cdot m_{k}\right)^{-1 / 2}}-m_{k}\right]}_{\eta_{k}(t)} \underbrace{\frac{1}{q-k} \sum_{i=k+1}^{q} \ell_{i}}_{\ell_{k}^{a v}}
$$

where $\ell_{k}^{a v}$ is the average of the $m_{k}(=q-k)$ lowest eigenvalues, $\eta_{k}(t)$ is the prediction factor and $t$ is a two-direction threshold that represents the confidence interval of the averaged eigenvalue. The eigenvalue $\ell_{k}$ is considered in the noise subspace when it satisfies the following condition

$$
\ell_{k} \leq \ell_{k}^{u p}
$$

\section{B. Predicted Eigenvalues Threshold Based Spectrum Sensing}

The PET method is employed for detecting the communications sources number. Another well-known approach to solve this problem is the ITC, and in particular minimum description length (MDL) and Akaike information criterion (AIC) [17]. The performance of PET method is superior to the MDL under low SNR and enjoys consistency under high SNR (contrary to the AIC) [18]. The PET method is based on (12) and consists in adaptively modeling the noise eigenvalues increase to determine the dimension of the noise subspace, and hence, the signal subspace dimension. Actually, the PET model is controlled by a single parameter $t$.

Spectrum sensing problem is a special case of the sources number detection one. In the absence of PUs, the estimated number of source signals should be zero. The original PET (OPET) method, as described above, can applied directly to conduct spectrum sensing. Based on that, the two hypotheses in (1) are reformulated as

$$
\begin{aligned}
& \mathcal{H}_{0}: \ell_{k} \leq \ell_{k}^{u p}, k=1,2, \cdots, q-1 \\
& \mathcal{H}_{1}: \hat{k} \geq 1, \hat{k}=\arg \max _{k=1, \cdots, q-1} \ell_{k}>\ell_{k}^{u p} .
\end{aligned}
$$

In the presence of $P$ primary users, the dimension of signal subspace is $P L+\sum_{j=1}^{P} C_{j}$ (i.e., the contribution of $P U_{j}$ is equivalent to $L+C_{j}$ eigenvalues). The idea is to detect at least one PU under $\mathcal{H}_{1}$. Since $C_{j}$ is unknown and difficult to be estimated, the presence of PUs is reflected by at least $L$ signal eigenvalues.

It is clear from (8) that when a certain eigenvalue $\lambda_{\hat{k}}$ belongs to the signal subspace, then $\lambda_{1}, \cdots, \lambda_{\hat{k}}$ are all signal eigenvalues. This leads to the fact that when $\ell_{1}$ is noise eigenvalue then $\mathcal{H}_{0}$ is detected, i.e., there is no signal when every eigenvalue does not exceed its own predicted threshold. Also, $\mathcal{H}_{1}$ is detected when $\ell_{L}$ correspond to signals. Therefore, the spectrum sensing problem as defined in (1) is modified to

$$
\begin{aligned}
\mathcal{H}_{0} & : \ell_{1} / \ell_{1}^{a v} \leq \eta_{1}(t), \\
\mathcal{H}_{1,0} & : \ell_{1} / \ell_{1}^{a v}>\eta_{1}(t), \\
\mathcal{H}_{1,1} & : \ell_{L} / \ell_{L}^{a v}>\eta_{L}(t) .
\end{aligned}
$$

where $\ell_{k}^{a v}$ is defined in (11). The $\mathcal{H}_{1,0}$ hypothesis indicates the presence of a weak PU's signal, while $\mathcal{H}_{1,1}$ is the sensing state for the presence of relatively non-weak signal. This method is called the modified PET (MPET). This extra information is sent to the media access control (MAC) layer of the cognitive radio and could be very beneficial for the adaptive joint scheduling of spectrum sensing and data transmission in cognitive radio networks. This research work is in our perspectives.

\section{Simplified Predicted Eigenvalues Threshold (SPET) METHOD}

The above discussion indicates that the PET method could be simplified to employ only one eigenvalue. The question is which eigenvalue $\ell_{\hat{k}}, 1 \leq \hat{k} \leq L$, must be employed for the spectrum sensing problem? It is $\ell_{1}$. In fact, when $\ell_{1}$ corresponds to noise, we are confident that all eigenvalues belong to the noise subspace. On the other hand, if $\ell_{1}$ is a signal eigenvalue, the PU detection must not be missed even though all the other eigenvalues corresponds to noise. This is due to the privilege of protecting PUs over spectrum utilization efficiency. Then, the PET method is simplified to test the largest eigenvalue against its own predicted threshold. Hence, the sensing problem can be expressed as $\ell_{1} / \ell_{1}^{a v} \underset{\mathcal{H}_{0}}{\stackrel{\mathcal{H}_{1}}{\gtrless}} \eta_{1}(t)$, and is altered to:

$$
T_{\text {SPET }}=\frac{\ell_{1}}{\frac{1}{q} \sum_{i=1}^{q} \ell_{i}} \underset{\mathcal{H}_{0}}{\stackrel{\mathcal{H}_{1}}{\gtrless}} \gamma,
$$

This decision statistic was found in [15], [16] based on GLRT for a single source through memoryless channel. Our work extends this decision statistic to a more general scenario. The outline of the proposed SPET method is described by the following algorithm.

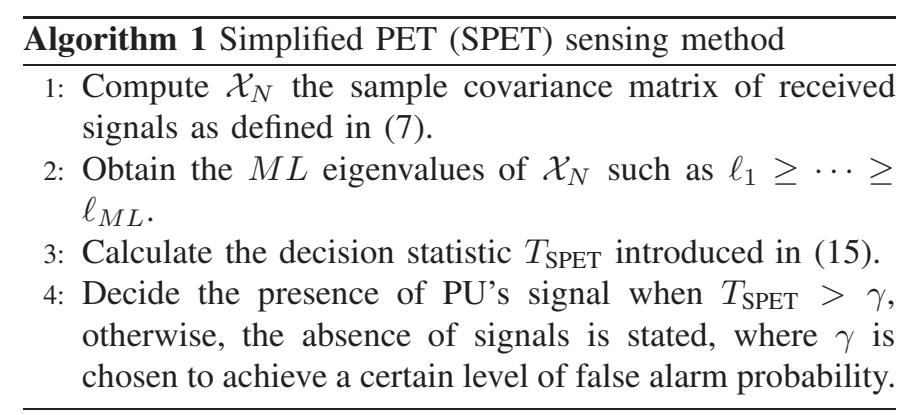

\section{Performance Analysis And Discussion}

Let $P_{m}=\mathbb{P}\left(\mathcal{H}_{0} \mid \mathcal{H}_{1}\right)$ be the probability of missing. The missed detection leads to harmful interference with existing PUs. The probability of false alarm is defined as $P_{f}=$ $\mathbb{P}\left(\mathcal{H}_{1} \mid \mathcal{H}_{0}\right)$. In fact false alarm error leads to inefficient usage 
of the spectrum. Practically, $P_{m}$ must be minimal while $P_{f}$ is kept less than a certain level.

The performance of OPET method is controlled by $t$. The two-direction threshold $t$ is chosen to satisfy a certain level of the false alarm probability $P_{f}$. For more details on the expressions of $P_{m}$ and $P_{f}$ of OPET refer to [18]. Here, we will focus on performance analysis of SPET. It is difficult to obtain a simple closed form expression for the distribution of $T_{\text {SPET }}$. This is due to the same difficulty regarding the largest eigenvalue.

\section{A. Probability of False Alarm}

New results in random matrix theory (RMT) revealed that $\ell_{1}$ converges in distribution to a Tracy-Widom distribution of order $\beta$ ( $\beta=1,2$ for real or complex observations, respectively) when $\mathcal{X}_{N}$ follows a Wishart distribution of order $N$ [20]. These results were extended in [21] for a more general class of samples. In their settings, the population model have a spectra similar to that employed in our paper. The authors in [16] established that these results imply that asymptotically $T_{\text {SPET }}$ also follows a Tracy-Widom distribution.

Theorem 2: Let $\mathcal{X}_{N}$ follows a Wishart distribution with parameters $N, q$ and $T_{\mathrm{SPET}}$ be the ratio of largest eigenvalue to the average trace. Then, as $N, q \rightarrow \infty$, with $c_{N}=\frac{q}{N} \rightarrow c \geq 0$, the following holds

$$
\mathbb{P}\left[\frac{T_{\mathrm{SPET}}-\mu_{N, q}}{\sigma_{N, q}}<\gamma\right] \approx F_{\beta}(\gamma)-\frac{1}{\beta q N}\left(\frac{\mu_{N, q}}{\sigma_{N, q}}\right)^{2} F_{\beta}^{\prime \prime}(\gamma)
$$

where the centering and scaling constants are

$$
\begin{aligned}
\mu_{N, q} & =\left(1+\sqrt{c_{N}}\right)^{2}, \\
\sigma_{N, q} & =N^{\frac{-2}{3}}\left(1+\sqrt{c_{N}}\right)\left(1+\frac{1}{\sqrt{c_{N}}}\right)^{1 / 3},
\end{aligned}
$$

and $F_{\beta}($.$) is the cumulative distribution function (CDF) of the$ Tracy-Widom law of order $\beta$, while $F_{\beta}^{\prime \prime}$ denotes the second derivate of $F_{\beta}$.

Proof: This claim descend from the work in [16], later extended by Nadler [22].

In [22], it was established that this approximation is accurate for finite value of $N$ and $q$, even when $N \gg q$. Hence, the probability of false alarm is given by

$$
\begin{aligned}
P_{f} & =\mathbb{P}\left[T_{\text {SPET }}>\gamma \mid \mathcal{H}_{0}\right] \\
& =1-F_{\beta}\left(\frac{\gamma-\mu_{N, q}}{\sigma_{N, q}}\right)+\frac{1}{\beta q N}\left(\frac{\mu_{N, q}}{\sigma_{N, q}}\right)^{2} F_{\beta}^{\prime \prime}\left(\frac{\gamma-\mu_{N, q}}{\sigma_{N, q}}\right)
\end{aligned}
$$

Now for a given $P_{f}$, the threshold $\gamma$ is obtained based on (18). Obviously, the decision threshold is independent of the noise power and the channel gains, and depends only on $N, q$ and $P_{f}$.

\section{B. Probability of Missing}

The sample covariance matrix is no longer a Wishart matrix in the presence of a signal. Here, we try to approximate the probability of missing in the presence of single strong source, i.e., the signal subspace contains an eigenvalue of multiplicity $L$. Under this assumption, it is clear that $\frac{1}{q} \sum_{i=1}^{q} \ell_{i}=$ $\frac{1}{q}\left[L \ell_{1}+\sum_{i=L+1}^{q} \ell_{i}\right]$ and the probability of missing is given

$$
\begin{aligned}
P_{m} & =\mathbb{P}\left[T_{\text {SPET }}<\gamma \mid \mathcal{H}_{1}\right] \\
& =1-\mathbb{P}\left[\frac{\ell_{1}}{\sigma_{w}^{2}}>\frac{(q-L) \gamma}{q-L \gamma} \xi\right]
\end{aligned}
$$

where $\xi=\frac{\frac{1}{q-L} \sum_{i=L+1}^{q} \ell_{i}}{\sigma_{w}^{2}}$ is asymptotically approximated to 1 [15]. Also the largest eigenvalue has a limiting Gaussian distribution as follows [23]

$$
\ell_{1} \stackrel{\text { dist }}{\longrightarrow} \mathcal{N}\left(\lambda_{1}+\frac{(q-L) \lambda_{1} \sigma_{w}^{2}}{N\left(\lambda_{1}-\sigma_{w}^{2}\right)}, \frac{\lambda_{1}^{2}}{N}\right),
$$

which imply that

$$
\frac{\ell_{1}}{\sigma_{w}^{2}} \stackrel{\text { dist }}{\longrightarrow} \mathcal{N}\left(\mu_{n}, \sigma_{n}^{2}\right),
$$

where $\mu_{n}=(1+\alpha \rho)\left(1+\frac{q-L}{\alpha \rho N}\right), \sigma_{n}^{2}=\frac{(1+\alpha \rho)^{2}}{N}, \alpha=\frac{q}{q-L}$ and $\rho$ is the instantaneous received SNR. Hence, $P_{m}$ is approximated as

$$
P_{m}=1-\mathrm{Q}\left(\frac{(q-L) \gamma}{(q-L \gamma) \sigma_{n}}-\frac{\mu_{n}}{\sigma_{n}}\right)
$$

The average probability of missing is computed by averaging over the distribution of $\rho$.

\section{RESUlTS AND DiscUSSION}

Here, we present some simulations results to demonstrate the effectiveness of the proposed sensing methods. These methods are evaluated through the probability of missing at a false alarm probability of $P_{f}=0.1$. In order to determine the threshold for this given $P_{f}$, the decision statistic is calculated for $10^{5}$ independent random trials (at $\mathcal{H}_{0}$, when there is no signal). We sort the decision statistic values in descending

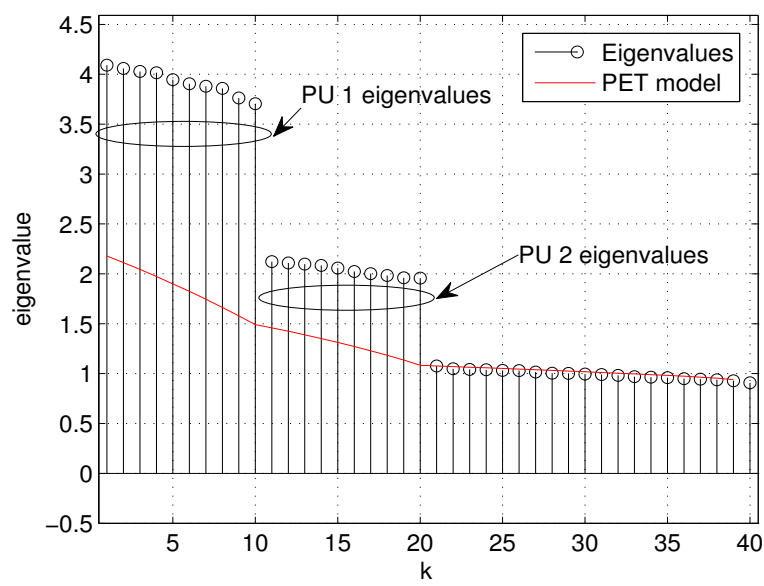

Figure 1. The eigenvalues and the adaptive PET model at $\mathrm{SNR}=0 \mathrm{~dB}$, $N=1000, P=2, M=4$ and $L=10$. 


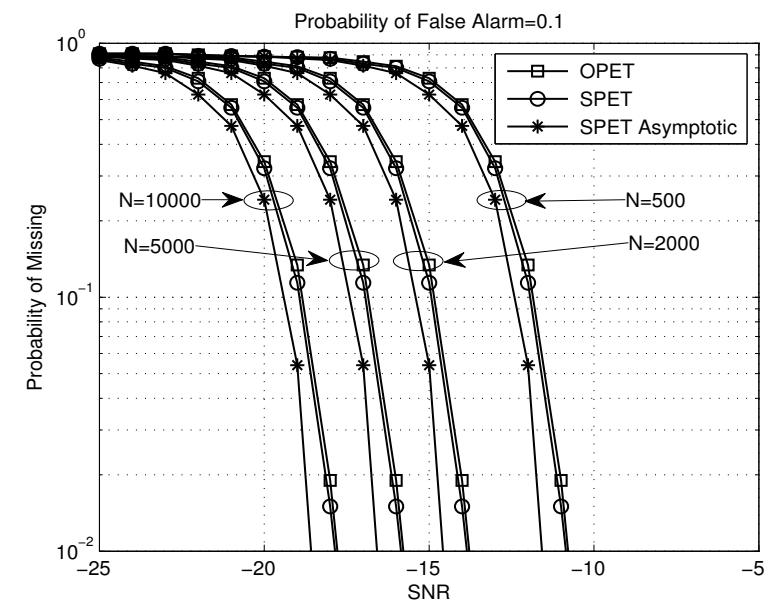

Figure 2. The probability of missing versus SNR for OPET and SPET methods compared with the asymptotic results at different values of the number of the observed samples $(\mathrm{N}=500,2000,5000$, and 10000) when $M=4, L=1$ and $P=1$.

order to choose the detection threshold such as $P_{f} \times 10^{5}$ samples of the generated statistic (out of $10^{5}$ ) are above the chosen threshold.

All results are based on 1000 Monte Carlo trials for each method. For each realization, the binary phase-shift keying modulated PU signals are randomly generated. Also, The random channel taps follow a Gaussian distribution. For different values of SNR, a random additive white Gaussian noise is added such that

$$
\mathrm{SNR}=\frac{E\left[\|\mathbf{x}(n)-\mathbf{w}(n)\|^{2}\right]}{E\left[\|\mathbf{w}(n)\|^{2}\right]}
$$

Fig. 1 shows the adaptive PET model of the sample covariance matrix eigenvalues at $\mathrm{SNR}=0 \mathrm{~dB}, P=2, M=4$ and $L=10$. It is clear that the noise subspace dimension is $(M-P) L$, while each PU is represented by $L$ eigenvalue ( $P L$ in total). Each signal eigenvalue exceeds its own predicted threshold.

Fig. 2 compares the sensing performance of OPET and SPET with the asymptotic results in (18) and (22). These simulations are done for different values of $N$ when $M=4$, $P=1$ and $L=1$. Based on simulations, the two-direction threshold $t$ is chosen 1.108 to achieve a false alarm probability of 0.1 . It is clear that the SPET method simplifies the original one to reduce the complexity without leading to any performance loss. Also, the simulations reveal that the asymptotic results provides a good approximation of the SPET sensing performance. Comparing the probability of missing for different values of $N$ shows that the performance improves when the number of the observed samples at the receiver is larger.

In Fig. 3 the probability of missing versus SNR for SPET method at different smoothing factor values $(L=1,5,8$, 10 and 15) is shown (for $N=10000, M=4, P=2$ and $\left.C_{1}=C_{2}=6\right)$. As can be seen from Fig. 3 increasing

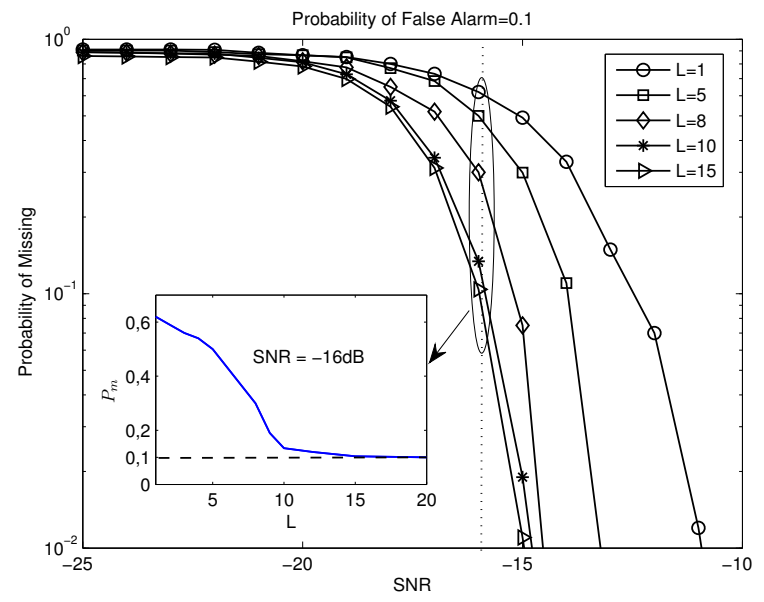

Figure 3. The probability of missing versus SNR for SPET algorithm at different smoothing factor values ( $\mathrm{L}=1,5,8,10$ and 15) when $N=10000$, $M=4, P=2$ and $C_{1}=C_{2}=6$. Also, the probability of missing versus $\mathrm{L}$ at a fixed $\mathrm{SNR}=-16 \mathrm{~dB}$

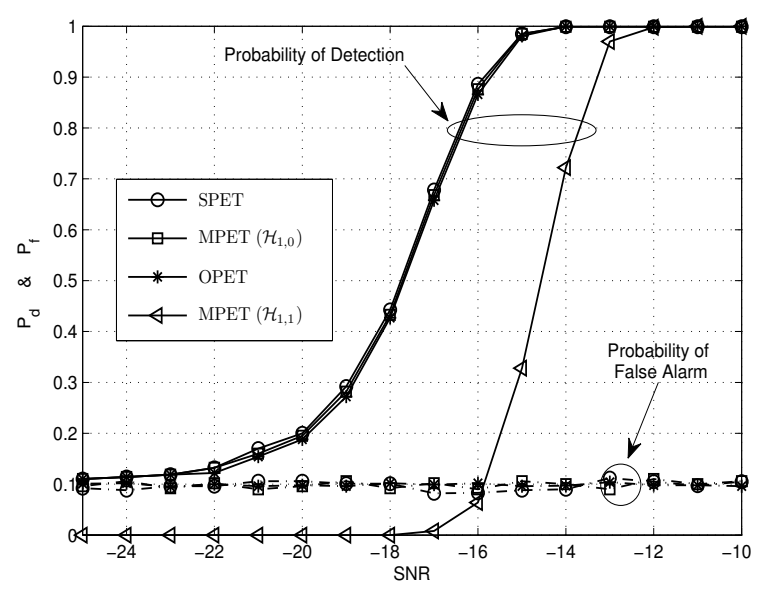

Figure 4. The MPET sensing performance (under $\mathcal{H}_{1,0}$ and $\mathcal{H}_{1,1}$ ) compared with OPET and SPET methods when $N=10000, M=4, P=2, L=10$ and $C_{1}=C_{2}=6$.

the smoothing factor up to 10 does improve the detection performance. But, the probability of missing is almost constant when $L>10$. In fact, the computational complexity increases when $L$ is larger. Hence, the smoothing factor is chosen relatively low as a compromise between decreasing complexity and not leading to a performance degradation. Moreover, we note that the SPET method still detects the presence of a signal even when $L$ does not satisfy the condition in (9), i.e. the results shown in Fig. 3 for $L=1,5$.

The performance of MPET method is illustrated in Fig. 4. This performance is evaluated by the detection probability of weak signals and that of relatively non-weak signals, set to as $\mathbb{P}\left(\mathcal{H}_{1,0} \mid \mathcal{H}_{1}\right)$ and $\mathbb{P}\left(\mathcal{H}_{1,1} \mid \mathcal{H}_{1}\right)$ respectively. This figure depicts the probability of detection and the false alarm probability of MPET, SPET and OPET methods. The detection probability of a weak signal is very close to that of SPET and OPET. On 


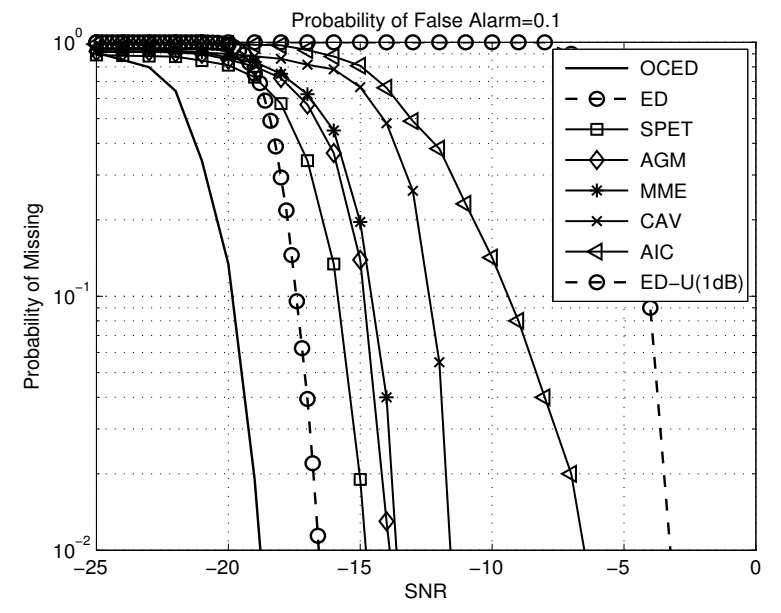

Figure 5. Probability of missing versus SNR for several sensing methods when $N=10000, M=4, P=2, C_{1}=C_{2}=6$ and $L=10$.

the other hand, the presence of relatively non-weak signal is stated with a detection probability of 0.9 when the SNR equals $-13 \mathrm{~dB}$

A performance comparison among several sensing methods is provided in Fig. 5. This comparison is done for the following parameters: $N=10000, M=4, P=2, C_{1}=C_{2}=6$ and $L=10$. The OCED [11] outperforms the ED as it maximizes the SNR. But this method is not blind since its decision statistic is $\frac{\lambda_{1}}{\sigma_{w}^{2}}$ [11]. The ED suffers of noise uncertainty. The noise power estimation error (in $\mathrm{dB}$ ) is assumed to be uniformly distributed in the interval $[-B, B][6]$, i.e., the $\mathrm{ED}$ is denoted "ED-U $(B \mathrm{~dB})$ ". It is clear that the performance significantly degrades when $B=1 \mathrm{~dB}$. Among the different blind methods the SPET one has the best performance followed by the AGM method [14]. This result is well expected since the two methods are GLRT based, but the SPET employs more the signal structure. The MME detection [13] is a bit less effective than the AGM detector but outperforms the CAV detector [12]. The CAV detection is directly based on the entries of the sample covariance matrix. Therefore, This method has a smaller complexity but it is less representative of the signals. Even though the ITC based methods [17] offer a low false alarm probability, they are, surprisingly, not as efficient as the other blind methods.

\section{CONCLUSIONS}

In this paper, the PET method, originally used for number of communications sources detection, is employed for multiple-antenna spectrum sensing. This method is simplified to significantly reduce the computational complexity without any performance loss compared with the original PET. The detection performance is well predicted by the asymptotic analysis which is based on new results in random matrix theory. The SPET test statistic generalizes that of the GLRT [15] to a more general scenario. The blind non-parametric SPET detector outperforms the other blind detectors existing in the literature.

\section{REFERENCES}

[1] "Spectrum policy task force," Federal Communications Commission, ET Docket No. 02-135, November 2002.

[2] J. Mitola, "Cognitive radio," Ph.D. dissertation, Roy. Inst. Technol., Stockholm, Sweden, 1999.

[3] C. R. Stevenson, C. Cordeiro, E. Sofer, and G. Chouinard, "Functional requirements for the 802.22 wran standard," IEEE Std. 802.2205/0007r46, Sep. 2005.

[4] D. Cabric, S. Mishra, and R. Brodersen, "Implementation issues in spectrum sensing for cognitive radios," in Asilomar Conf. on Signals, Systems and Computers, vol. 1, Pacific Grove, California, USA, Nov. 2004, pp. 772-776.

[5] H. Urkowitz, "Energy detection of unkown deterministic signals," Proceedings of the IEEE, vol. 55, no. 4, pp. 523-531, 1967.

[6] R. Tandra and A. Sahai, "Snr walls for signal detection," IEEE Journal of Selected Topics in Signal Processing, vol. 2, no. 1, pp. 4-17, Feb. 2008.

[7] J. Lundén, S. A. Kassam, and V. Koivunen, "Robust nonparametric cyclic correlation based spectrum sensing for cognitive radio," IEEE Transactions on Signal Processing, vol. 58, no. 1, pp. 38-52, January 2010.

[8] Y. H. Zeng, Y.-C. Liang, A. T. Hoang, and R. Zhang, "A review on spectrum sensing for cognitive radio: Challenges and solutions," EURASIP Journal on Advances in Signal Processing, pp. 1-16, Oct. 2010.

[9] A. Pandharipande and J. M. G. Linnartz, "Performance analysis of primary user detection in a multiple antenna cognitive radio," in IEEE Int. Conf. on Commun. (ICC 2007), Jun. 2007, pp. 6482-6486.

[10] J. Ma, G. Zhao, , and Y. Li, "Soft combination and detection for cooperative spectrum sensing in cognitive radio networks," IEEE Transactions on Wireless Communications, vol. 11, no. 7, pp. 4502-4507, Nov. 2008.

[11] Y. H. Zeng, Y.-C. Liang, and R. Zhang, "Blindly combined energy detection for spectrum sensing in cognitive radio," IEEE Signal Processing Letters, vol. 15, pp. 649-652, Oct. 2008.

[12] Y. H. Zeng and Y.-C. Liang, "Spectrum-sensing algorithms for cognitive radio based on statistical covariances," IEEE Transactions on Vehicular Technology, vol. 58, no. 4, pp. 1804-1815, 2009.

[13] _ - "Eigenvalue-based spectrum sensing algorithms for cognitive radio," IEEE Transactions on Communications, vol. 57, no. 6, pp. 1784 1793, 2009.

[14] R. Zhang, T. J. Lim, Y.-C. Liang, and Y. Zeng, "Multi-antenna based spectrum sensing for cognitive radios: A glrt approach," IEEE Transactions on Communications, vol. 58, no. 1, pp. 84-88, Jan. 2010.

[15] A. Taherpour, M. Nasiri-Kenari, and s. Gazor, "Multiple antenna spectrum sensing in cognitive radios," IEEE Transactions on Wireless Communications, vol. 9, no. 2, pp. 814-823, Feb. 2010.

[16] P. Bianchi, M. Debbah, M. Maida, and J. Najim, "Performance of statistical tests for single-source detection using random matrix theory," IEEE Transactions on Information theory, vol. 57, no. 4, pp. 1-20, April 2011.

[17] R. Wang and M. Tao, "Blind spectrum sensing by information theoretic criteria for cognitive radios," IEEE Transactions on Vehicular Technology, vol. 59, no. 8, pp. 3806-3817, Oct. 2010.

[18] W. Chen, K. M. Wong, and J. P. Reilly, "Detection of the number of signals: A predicted eigen-threshold approach," IEEE Transactions on Signal Processing, vol. 39, no. 5, pp. 1088-1098, 1991.

[19] E. Moulines, P. Duhamel, J. F. Cardoso, and S. Mayrargue, "Subspace methods for the blind identification of multichannel fir filters," IEEE Transactions on Signal Processing, vol. 43, no. 2, pp. 516-525, Feb. 1995.

[20] I. Johnstone, "On the distribution of the largest eigenvalue in principal components analysis," The Annals of Statistics, vol. 29, no. 2, pp. 295$327,2001$.

[21] J. Baik and J. W. Silverstein, "Eigenvalues of large sample covariance matrices of spiked population models," Journal of Multivariate Analysis, vol. 97, no. 6, pp. 1382-1408, July 2006.

[22] B. Nadler, "On the distribution of the ratio of the largest eigenvalue to the trace of a wishart matrix," Journal of Multivariate Analysis, vol. 102, no. 2, pp. 363-371, Feb. 2011.

[23] F. Haddadi, M. M. Mohammadi, M. M. Nayebi, and M. R. Aref, "Statistical performance analysis of mdl source enumeration in array processing," IEEE Transactions on Signal Processing, vol. 58, no. 1, pp. 452-457, Jan. 2010. 